\title{
Letter from the guest editors of the topical collection on satellite orbit determination
}

\author{
Tzu-Pang Tseng ${ }^{1} \cdot$ Pieter N. A. M. Visser ${ }^{2}$
}

Published online: 18 December 2021

(c) The Author(s), under exclusive licence to Springer-Verlag GmbH Germany, part of Springer Nature 2021

This Topical Collection (TC) focuses on satellite orbit determination, mainly supporting a wide range of space objects using ground-based tracking systems, such as the Global Navigation Satellite System (GNSS), Satellite Laser Ranging (SLR) and Doppler Orbitography and Radiopositioning Integrated by Satellite (DORIS). For a high-orbit navigation satellite, the orbit is a reference datum for its ranging measurement, used for positioning, navigation, and timing applications. As to low earth orbit (LEO) satellites for earth observation, the orbital information serves as a probe for detecting or sensing large-scale geodynamic and geophysical signals, e.g., observing mass transport, sea level rise, glaciology, etc. In addition, micro-satellite constellations autonomous orbital operation and inter-satellite ranging measurements are also targeted for exploring new opportunities in space.

Satellite orbit determination essentially consists of (1) detailed force models, (2) availability of ground-based tracking systems, (3) high-precision time and frequency standards, (4) high-accurate station coordinates in connection to a well-defined reference frame, and (5) parameter definition and estimation methods. Errors in each component may bias the orbit solution to some extent. Nowadays, the accuracy of the satellite orbit determination is mainly limited by the following areas: (1) non-gravitational force modeling, e.g., atmospheric drag, solar radiation pressure, earth radiation pressure, and antenna thrust, (2) satellite attitude knowledge, (3) time-variable gravity field, (4) antenna phase center

Tzu-Pang Tseng

tzupangtseng@nkust.edu.tw

1 Department of Civil Engineering, National Kaohsiung University of Science and Technology, No. 415, Jiangong Rd., Sanmin Dist., Kaohsiung City 80778, Taiwan

2 Faculty of Aerospace Engineering, Delft University of Technology, Kluyverweg 1, 2629 HS Delft, The Netherlands correction, (5) ionospheric activity, and (6) tracking data quality associated with the clock stability.

The TC on Satellite Orbit Determination started in December 2020, at about the same time as other collections were introduced. Authors can assign the paper to a topical collection at the time of paper submission. The Editor In Chief initially checks all papers submitted, even those not assigned to a collection, to see if they should be assigned to a collection or treated as a non-topical-collection paper and takes respective action. Once the Editor In Chief has approved the assignment to a collection, the respective guest editor(s) initially check the papers. If a paper fits the scope of the collection and is judged to have a potential for eventual publication, a scientific review is carried out by two anonymous reviewers. If it does not fit the scope of the topical collection, the manuscript is returned to the Editor In Chief for further considerations. Based on this process, 6 papers have been accepted so far.

The first paper entitled "Comparisons of CODE and CNES/CLS GPS satellite bias products and applications in Sentinel-3 satellite precise orbit determination" is published by Duan and Hugentobler. The authors compared the satellite bias products from CNES/CLS (Center National d'Etudes Spatiales/ Collecte Localisation Satellites) to those from CODE (Center for Orbit Determination in Europe) and used both of them for precise orbit determination (POD) of Sentinel-3 satellite. The authors found that the satellite bias from CNES/CLS is tightly constrained to the solution from previous day, whereas that from CODE varies from day to day. With the satellite bias, the ambiguity-fixed POD solution of the Sentinel-3 satellite shows a significant improvement over the ambiguity-float one by $10 \%$ for Sentinel-3A and $15 \%$ for Sentinel-3B when SLR measurement is used for orbit validation.

The second paper entitled "GPS, GLONASS and Galileo orbit geometry variations caused by general relativity focusing on Galileo in eccentric orbits" is published by Sośnica et al. The general relativity effect such as the Schwarzschild, 
Lense-Thirring, and De Sitter effects may lead to changes in the satellite orbit geometry and orientation. The authors estimate the post-Newtonian parameters using the satellite orbit determination technique with three years of real GNSS tracking data and discover the impact of the general relativity on orbital parameters, such as the semimajor axis and the eccentricity. The mean semimajor axis offset of GPS, GLONASS, and Galileo satellites is $-17.41 \mathrm{~mm}$, which is slightly larger than the theoretical value by $0.36 \%$.

The third paper entitled "Performance assessment of GNSS-based real-time navigation for Sentinel-6 spacecraft" is published by Montenbruck et al. The authors use onboard flight data, including carrier phase measurements from GPS + Galileo and broadcast ephemeris from the Sentineal$6 \mathrm{~A}$ for the precise real-time orbit determination as a navigation solution. Such a navigation solution is determined with a positioning error of $10 \mathrm{~cm}$ (3D RMS), which is similar to that given by the DORIS navigation system. This work will be feasible for future remote sensing missions.

The fourth paper entitled "Precise orbit determination of BDS-3 satellites using B1C and B2a dual-frequency measurements" is published by $\mathrm{Li}$ et al. The results show that the BDS-3 orbit solution using both B1C and B2a is better than that using both B1I and B3I by $9 \%$ in terms of orbit overlaps. Furthermore, the authors also demonstrated that the ECOM SRP model with 7 parameters $\left(D_{0}, D_{\mathrm{Cu}}, D_{\mathrm{Su}}, Y_{0}, B_{0}, B_{\mathrm{Cu}}\right.$ and $B_{\mathrm{Su}}$ ) presents a relatively good orbit solution as compared to those with $5\left(D_{0}, Y_{0}, B_{0}, B_{\mathrm{Cu}}\right.$ and $\left.B_{\mathrm{Su}}\right)$ and 9 parameters $\left(D_{0}\right.$, $D_{\mathrm{Cu}}, D_{\mathrm{Su}}, Y_{0}, Y_{\mathrm{Cu}}, Y_{\mathrm{Su}}, B_{0}, B_{\mathrm{Cu}}$ and $\left.B_{\mathrm{Su}}\right)$.

The fifth paper entitled "Assessment of single-difference and track-to-track ambiguity resolution in LEO precise orbit determination" is published by Zhou et al. The authors assessed the ambiguity resolution via the single-difference (SD) and track-to-track (T2T) strategies with three months of GRACE-FO tracking data. The authors found that it is highly reliant on the variations of receiver hardware delays in the T2T case, while Fractional Cycle Bias (FCB) or Integer Recovery Clock (IRC) products are required in the SD case. The SD-derived orbit solution is similar to the T2Tderived one in the so-called reduced-dynamic orbit determination, while the SD-derived solution is slightly better than the T2T-derived one in the kinematic orbit determination.

The sixth paper entitled "Determination of precise Galileo orbits using combined GNSS and SLR observations" is authored by Bury et al. An approach for GNSS-SLR combination with three different weighting strategies was developed. Such an approach is used to assess the impact of SLR observations on the determined Galileo orbits. The GNSS-SLR combination solution reduces the formal error of the semi-major axis by about $62 \%$ and also reduces the dependency between the formal error and the sun elevation angle with respect to the orbit plane. Besides, the drift of length-of-day parameter is improved by $20 \%$ in the combination case as compared to the GNSS-only case.

We would like to thank all reviewers for their valuable comments on the papers. Last but not least, we are grateful for your interest in this GPS Solutions Topical Collection of Satellite Orbit Determination.

Publisher's Note Springer Nature remains neutral with regard to jurisdictional claims in published maps and institutional affiliations.

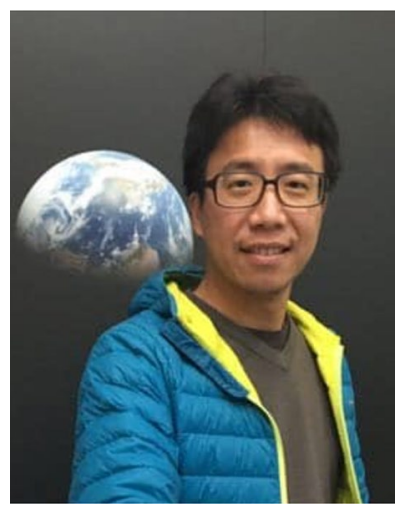

Dr. Tzu-Pang Tseng received his Ph.D. degree from the Department of Civil Engineering, National Chiao Tung University, Taiwan, in 2010. He is now an Assistant Professor at the Department of Civil Engineering, National Kaoshiung University of Science and Technology (NKUST), Taiwan. Since 20172021, he was working as a POD (precise orbit determination) scientist at Geoscience Australia for developing Australian selfcontained GNSS software, called Ginan. In this position, he was in charge of the non-gravitational force modeling on satellite orbit determination. In addition, he was leading POD projects in the FORMOSAT-3/COSMIC and FORMOSAT-7/COSMIC-2 satellite missions at National Central University, Taiwan, since 2012 to 2017. His research topic focus on the satellite orbit determination, satellite clock estimation and GNSS-based positioning applications.

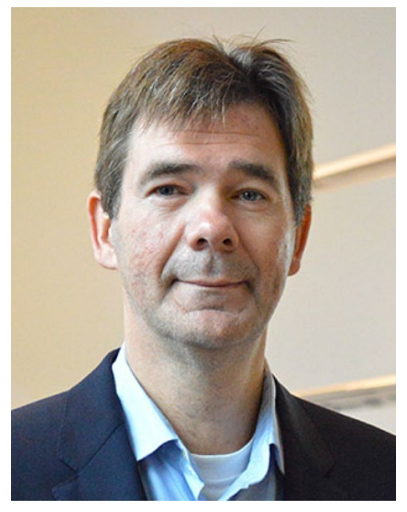

Prof. dr. ir. Pieter N. A. M. Visser Pieter Visser is head of the Department of Space Engineering at the Faculty of Aerospace Engineering of the Delft University of Technology in the Netherlands, where he holds the chair of Astrodynamics and Space mission. His primary fields of expertise are precise orbit determination of satellites and space geodesy. After receiving his $\mathrm{PhD}$ degree in 1992 in Delft, he spent one year as exchange visitor at the Center for Space Research of the University of Texas at Austin. He then returned to his Alma Mater. Over the years he has been involved in several earth observation missions aiming at observing sea level change, melting ice caps, gravity and magnetic fields. He has held many mission and science advisory positions for the European Space Agency (ESA), and several positions for the International Association of Geodesy (IAG). He chaired the COSPAR Panel on Satellite Dynamics from 2004 to 2012. He is Fellow of the IAG and full member of the International Academy of Astronautics (IAA). 